\title{
Automatically Gradient Threshold Estimation of Anisotropic Diffusion for Meyer's Watershed Algorithm Based Optimal Segmentation
}

\author{
Mithun Kumar PK", Md. Gauhar Arefin, Mohammad Motiur Rahman and A. S. M. Delowar Hossain \\ Dept. of Computer Science \& Engineering, Mawlana Bhashani Science and Technology University, Tangail-1902, \\ Dhaka, Bangladesh \\ Email: \{“mithun24bd, garefin005, mm73rahman\}@gmail.com, uzzal35@yahoo.com
}

\begin{abstract}
Medical image segmentation is a fundamental task in the medical imaging field. Optimal segmentation is required for the accurate judgment or appropriate clinical diagnosis. In this paper, we proposed automatically gradient threshold estimator of anisotropic diffusion for Meyer's Watershed algorithm based optimal segmentation. The Meyer's Watershed algorithm is the most significant for a large number of regions separations but the over segmentation is the major drawback of the Meyer's Watershed algorithm. We are able to remove over segmentation after using anisotropic diffusion as a preprocessing step of segmentation in the Meyer's Watershed algorithm. We used a fixed window size for dynamically gradient threshold estimation. The gradient threshold is the most important parameter of the anisotropic diffusion for image smoothing. The proposed method is able to segment medical image accurately because of obtaining the enhancement image. The introducing method demonstrates better performance without loss of any clinical information while preserving edges. Our investigated method is more efficient and effective in order to segment the region of interests in the medical images indeed.
\end{abstract}

Index Terms-Anisotropic diffusion, Computed tomography, Gradient threshold, Medical image, Morphological operation, Segmentation, Watershed algorithm.

\section{INTRODUCTION}

2D Computed tomography (CT) can provide detailed anatomic information about the heart chambers, large vessels, and coronary arteries, brain tumor, collection of blood in a place compared with other imaging modalities such as ultrasonic (US) imaging, magnetic resonance imaging (MRI) etcetera [1]. Therefore, 2D CT is an important imaging modality for diagnosing cardio vascular, brain and so on diseases which require volume, shape, position and other parameters. The complete segmentation is the prerequisite for clinical investigations and providing critical information for quantitative functional analysis [2], [3].
In this paper, we proposed an automatic 2D segmentation system using a surface-based model. There are two major tasks to develop such a segmentation system of any organ modeling (shape representation) and edge detection as well as segmentation. A large number of mathematical morphology based image segmentation approaches [8-22] are found in the available literature. Due to the complexity of anatomy, it is not trivial to represent the anatomy accurately while keeping the model simple enough for segmentation and manual correction if necessary.

Diffusion based spatial filtering methods have been proposed in [23-32]. An edge sensitive diffusion method is introduced in [28] for edge detection and segmentation. However, anisotropic diffusion is a nonlinear diffusion approach to spatial adaptive filtering, which demonstrates effective results of image segmentation in homogeneous regions while preserves edges effectively and significantly. A tensor-based anisotropic diffusion method is delineated in [29].

Nowadays, image segmentation is the most important task in the signal processing field because of having bereft of the desired consequences with respect to the natural world crisis and problems. However, many techniques are proposed for optimal image segmentation, which techniques and methodologies are depicted in [3340] with lots of experimental results according to the specific fields. Here, we investigated a new approach in anisotropic diffusion for obtaining optimal segmentation in medical images.

A flexible model is necessary to capture the any shape at any appropriate level of details. Hence, our proposed method makes it possible to get the shape in a 2D CT image. The Meyer's Watershed algorithm [4] is the segmentation algorithm, which can segment CT image. But it does not able to find the correct and accurate shapes because of over segmentation.

We proposed a preprocessing technique oriented method, which provides us a smooth image. The gradient magnitude [41-44] is often used to preprocess a gray level image prior to put the image under watershed operation. We apply original Meyer's Watershed algorithm on that preprocessed smooth image to obtain desired shapes and edges. 
In the paper, we explain original Meyer's Watershed algorithm in section II. In section III we proposed our preprocessing technique and segmentation process. In section IV we depict experimental results by visual comparison. In section $\mathrm{V}$ we concluded this paper.

\section{MEYER's WATERSHEd ALGORITHM FOR SEGMENTATION}

Meyer's Watershed algorithm was introduced by $\mathrm{F}$. Meyer [4] in the early 90's, which is one of the most effective segmentation algorithms. The algorithm works on a gray scale image. During the successive flooding of the grey value raised area, watersheds with adjacent catchment basins are constructed. This flooding process is performed on the gradient image, i.e. the basins should connect adjacent edges. Generally, this algorithm will lead to an over-segmentation of the image, especially for artifacts or noises image material, e.g. medical CT data, Ultrasound image. Either the image must be preprocessed or the regions must be merged on the basis of a similarity criterion afterwards.

1) A set of markers, pixels where the flooding shall start are chosen. Each is given a different label.

2) The neighboring pixels of each marked area are inserted into a priority queue with a priority level corresponding to the gray level of the pixel.

3) The pixel with the highest priority level is extracted from the priority queue. If the neighbors of the extracted pixel that have already been labeled all have the same label, then the pixel is labeled with their label. All non-marked neighbors that are not yet in the priority queue are put into the priority queue.

4) Redo step 3 until the priority queue is empty. The non-labeled pixels are the watershed lines.

\section{PROPOSED METHOD}

The Watershed algorithm is more efficient for optimal segmentation. This algorithm is used widely in medical imaging. Watershed algorithm performance depends on the ratio of homogeneous and edge regions. If we can properly select the edges in an image then watershed based segmentation will be optimized. Over segmentation is the major limitation of the watershed algorithm. For avoiding this limitation we need to preprocess the image for effective homogeneous region expansion. Here we used various preprocessing techniques with Meyer's Watershed algorithm for optimal segmentation. The proposed method is depicted step by step with mathematical explanations and parameters below:

\section{A. Preprocessing}

The preprocessing is the fundamental task for the desired segmentation in the case of watershed algorithms. It is mainly operated by convolution and anisotropic diffusion as well as especially morphological operation is used due to the reduction of over segmentation.

\section{a) Convolution}

The convolution is an investigated for smoothing of an image. Hence, we use Gaussian smoothing, which is defined as follows:

Let the grey-scale image $f$ be represented by a realvalued mapping $f \in L^{1}\left(\mathfrak{R}^{2}\right)$. A widely-used way to smooth $f$ by calculating the convolution

$$
\left(K_{\sigma}^{*} f\right)(x):=\int_{\Re^{2}} K_{\sigma}(x-y) f(y) d y
$$

where $K_{\sigma}$ denotes the two-dimensional Gaussian of width (standard deviation) $\sigma>0$ :

$$
K_{\sigma}(x):=\frac{1}{2 \pi \sigma^{2}} \cdot \exp \left(-\frac{|x|^{2}}{2 \sigma^{2}}\right)
$$

Here, we used $5 \times 5$ Gaussian kernel for the image smoothing. We know the standard deviation is the degree of the smoothness. Thus, a large standard deviation is ensured by the large kernel size, which is the most important parameter in order to smooth the image.

\section{b) Anisotropic Diffusion}

Perona and Malik[5], [6] proposed the following nonlinear PDE For smoothing image on a continuous domain:

$$
\left\{\begin{array}{l}
\frac{\partial I}{\partial t}=\operatorname{div}[g(|\nabla I|) \cdot \nabla I] \\
I(t=0)=I_{0}
\end{array}\right.
$$

where $\nabla$ is the gradient operator, div is the divergence operator, |.| denotes the magnitude, $g(||$.$) is the diffusion$ coefficient, and $I_{0}$ is the initial image. They suggested two diffusion coefficients

$$
g(x)=\frac{1}{1+(x / k)^{2}}
$$

and

$$
g(x)=\exp \left[-(x / k)^{2}\right]
$$

where $k$ is the edge magnitude parameter.

Here we used (4) for anisotropic coefficient measurement. The edge magnitude parameter or gradient threshold parameter $k$ is dynamically calculated by the fixed window size. We consider $5 \times 5$ window (Fig. 1) for appropriately estimating the value of $k$, which is essential parameter for meaningful blurring effect in an image. The value of $k$ is computed by the window size, the mean 
value and the standard deviation of this window covered pixel values.

\begin{tabular}{|c|c|c|c|c|}
\hline$I(i-2, j-2)$ & $I(i-1, j-2)$ & $I(i, j-2)$ & $I(i+1, j-2)$ & $I(i+2, j-2)$ \\
\hline$I(i-2, j-1)$ & $I(i-1, j-1)$ & $I(i, j-1)$ & $I(i+1, j-1)$ & $I(i+2, j-1)$ \\
\hline$I(i-2, j)$ & $I(i-1, j)$ & $I(i, j)$ & $I(i+1, j)$ & $I(i+2, j)$ \\
\hline$I(i-2, j+1)$ & $I(i-1, j+1)$ & $I(i, j+1)$ & $I(i+1, j+1)$ & $I(i+2, j+1)$ \\
\hline$I(i-2, j+2)$ & $I(i-1, j+2)$ & $I(i, j+2)$ & $I(i+1, j+2)$ & $I(i+2, j+2)$ \\
\hline
\end{tabular}

Fig.1. $5 \times 5$ window for edge magnitude parameter dynamically estimation.

For the gradient threshold parameter $k$, we used

$$
k=2 \log (n \times n) \frac{\sqrt{\mu}}{\sigma}
$$

where

$$
\mu=\frac{1}{n \times n} \sum_{x=-n / 2}^{n / 2} \sum_{y=-n / 2}^{n / 2} I(i+x, j+y)
$$

and

$$
\sigma=\sqrt{\frac{\sum_{x=-n / 2}^{n / 2} \sum_{y=-n / 2}^{n / 2}[I(i+x, j+y)-\mu]^{2}}{n \times n}}
$$

here, $\mu$ is the mean value, which is calculated for extracting the central tendency, $\sigma$ is the standard deviation, which is computed for measuring the signal magnitude fluctuation and $n$ is a positive integer number. We used $n=5$ for generating the optimal desirable result.

\section{c) Morphological Operation}

We applied reconstruction-based opening and closing for avoiding over segmentation, which are more effective than standard opening and closing [7] at removing small blemishes without affecting the overall shapes of an image Hence, we followed Fig. 2 for executing morphological operation in an image for obtaining the optimistic consequences. Overall, the morphological implementation sequence is followed by Fig. 2:

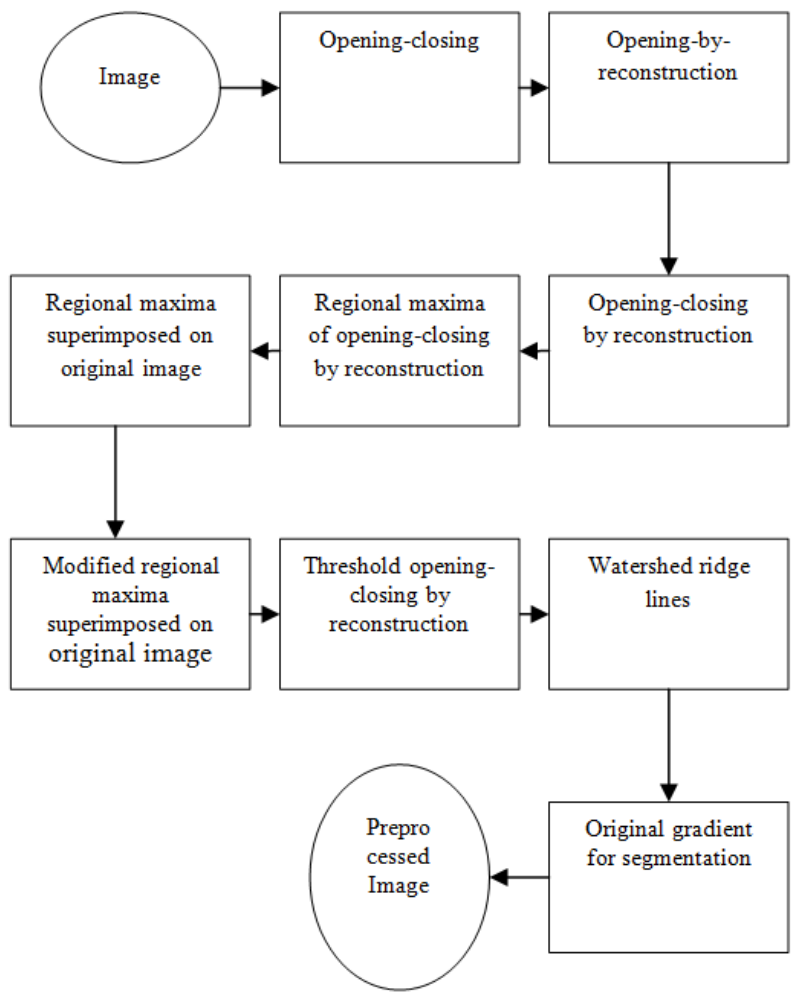

Fig.2. Morphological operation in sequence

\section{B. Segmentation}

We proposed preprocessing technique oriented method, which makes an image smooth as well as allows an image to avoid over segmentation. Because of the over segmentation, it is not absolutely possible to obtain the optimal segmentation of an image. Finally, we use original Meyer's Watershed algorithm [4] after receiving the preprocessed gradient image for the optimal segmentation.

\section{EXPERIMENTAL RESULTS}

For experimental validation, we visually compare between the Original Meyer's Watershed segmentation algorithm and the proposed optimal segmentation using Meyer's Watershed algorithm. We try to segments 2D CT image of the heart and brain image as well as to visualize the heart chambers. We performed all experiments in MATLAB 7.12.0.637 (R2011a) which in 32-bit (win32), processed in CORE-i5, RAM 4GB and in operating system Windows-7. Figs. 3 and 4 demonstrate the efficacy effectively of the proposed method. 
Visual comparisons are given below:

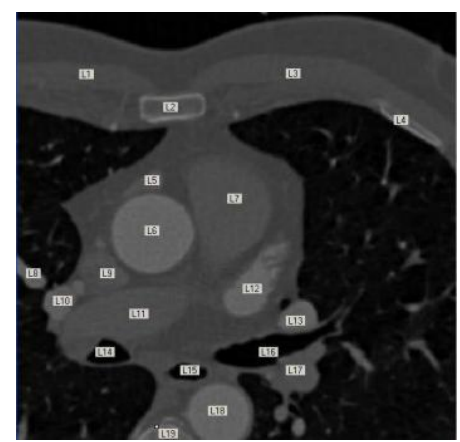

(a)

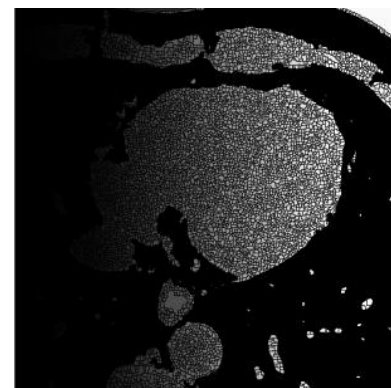

(b)

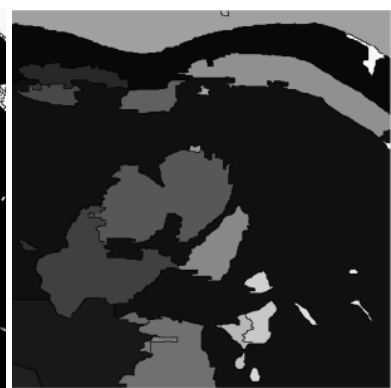

(c)
Fig. 3. 2D CT heart image, (a) Original image (b) Segmented image for General Meyer's Watershed algorithm and (c) Segmented image for proposed algorithm.

In Fig. 3(a), we used a 2D CT heart image which is in gray scale, 16 bits and 512 x 512 is the image size. We labeled different parts of the original image. L6, L7, L11, and L12 are the left atrium, left ventricle, right atrium and right ventricle respectively. Here, we tried to segment the chambers of the heart clearly and appropriately. From Fig. 3(b) we observe that the Meyer's Watershed algorithm develops over segmentation and unable to detect homogeneous structure and edge boundaries properly in an image but the Meyer's Watershed is more efficient for the optimal region separation with respect to the existing a state of the art segmentation algorithms. Fig. 3(c) is our proposed filtered result, which is obtained after executing anisotropic based preprocessing in Meyer's Watershed algorithm. The proposed filter is able to segment the heart chambers strongly.

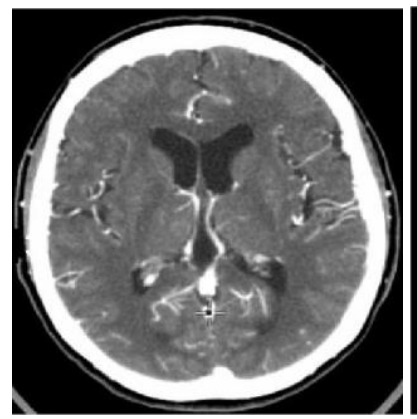

(a)

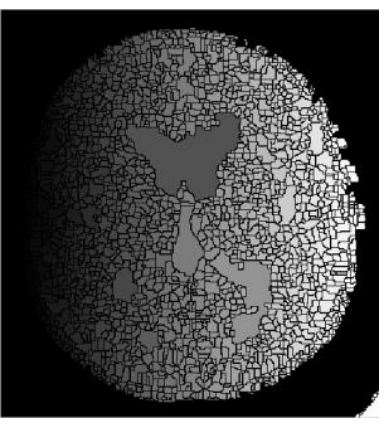

(b)

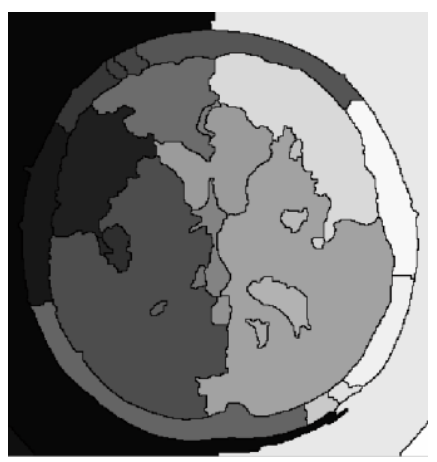

(c)

Fig. 4. 2D CT brain image, (a) Original image (b) Segmented image for General Meyer's Watershed algorithm and (c) Segmented image for proposed algorithm.

In Fig. 4(a) we used a 2D CT brain image which is in gray scale, 8 bits and $354 \times 396$ is the image size. From Fig. 4(b) we observe that Meyer's Watershed algorithm generates over segmentation and unable to detect homogeneous region and edge boundaries appropriately in an entire image as like as the heart image, but the Meyer's Watershed is more efficient to discriminate the optimal region with respect to the existing a state of the art segmentation algorithms. Fig. 4(c) is our proposed filtered image, which demonstrates imperative optimal segmentation as well as exhibits all of the portions clearly in the brain image. This proposed consequence is the most significant and efficient than the conventional Meyer's Watershed algorithm consequence for the clinical analysis and diagnosis.

\section{CONCLUSIONS}

The proposed approach is the most significant and effective for the optimal segmentation in the 2D CT medical images. The investigated method is fully designed by preprocessing approach. The preprocessing procedure mainly influenced by anisotropic diffusion, where the gradient threshold is estimated dynamically based on the local pixel grouping as well as the gradient threshold parameter depends on those pixels properties. However, we consider $5 \times 5$ window size for locally central pixel's diffusion for alleviating noises from the observe images. Finally, we apply the Meyer's Watershed algorithm on the smooth image in order to obtain the desired optimal segmented image.

\section{REFERENCES}

[1] P.Schoenhagen, S. S. Halliburton, A. E. Stillman, and R. D. White, "CT of the heart: Principles, advances, clinicaluses," Cleveland Clinic Journal of Medicine, vol.72, no.2, 2005, pp.127-138.

[2] F. Frangi, W. J. Niessen, and M. A. Viergever, "Threedimensional modeling for functional analysis of cardiac images: A review," IEEE Trans. Medical Imaging, vol.20, no.1, 2001, pp.2-25. 
[3] F. Frangi, D.Rueckert, and J. S. Duncan, "Threedimensional cardio vascular image analysis," IEEE Trans. Medical Imaging, vol.21, no.9, 2002, pp.1005-1010.

[4] Serge Beucher and Fernand Meyer. The morphological approach to segmentation: the watershed transformation. In Mathematical Morphology in Image Processing (Ed. E. R. Dougherty), pages 433-481 (1993).

[5] P. Perona, J.Malik, Scale space and edge detection using anisotropic diffusion, Proc. IEEE Comp. Soc. Workshop on Computer Vision (Miami Beach, Nov.30 -Dec.2, 1987), IEEE Computer Society Press, Washington, pp.16-22,1987.

[6] P. Perona, J. Malik, Scale space and edge detection using anisotropic diffusion, IEEE Trans. Pattern Anal. Mach. Intell., Vol.12, 1990, pp.629-639.

[7] Digital Image Processing by R. Gonzalez, Third Edition, 2005.

[8] S. Beucher and C. Lantuejoul, "Use of watersheds in contour detection," 1979.

[9] F. Meyer and S. Beucher, "Morphological segmentation," Journal of visual communication and image representation, vol. 1, pp. 21-46, 1990.

[10] L. Vincent, Algorithmes morphologiques a base de files d'attente et de lacets: extension aux graphes: Paris, 1990.

[11] F. Meyer, "Topographic distance and watershed lines," Signal Processing, vol. 38, pp. 113-125, 1994.

[12] A. N. Moga and M. Gabbouj, "Parallel image component labeling with watershed transformation," IEEE transactions on pattern analysis and machine intelligence, vol. 19, pp. 441-450, 1997.

[13] J. Roerdink and A. Meijster, "The watershed transform: Definitions, algorithms and parallelization strategies," Mathematical Morphology, vol. 41, pp. 187-S28, 2000.

[14] P. Soille, Morphological image analysis: principles and applications: Springer-Verlag New York, Inc. Secaucus, NJ, USA, 1999.

[15] J. Serra, Image analysis and mathematical morphology: Academic Press, Inc. Orlando, FL, USA, 1983.

[16] J. Serra and L. Vincent, "An overview of morphological filtering," Circuits, Systems, and Signal Processing, vol. 11, pp. 47-108, 1992.

[17] J. Roerdink and A. Meijster, "The watershed transform: Definitions, algorithms and parallelization strategies," Mathematical Morphology, vol. 41, pp. 187-S28, 2000.

[18] Angulo, J., Jeulin, D., "Stochastic watershed segmentation", in: Banon, G.J.F., Barrera, J., Braga-Neto, U.d.M., Hirata, N.S.T. (Eds.), Proceedings of the 8th International Symposium on Mathematical Morphology (ISMM 2007), Instituto Nacional de Pesquisas Espaciais (INPE), S ao Jos'e dos Campos. pp. 265-276, 2007.

[19] Jiann-Jone Chen, Chun-Rong Su, Grimson, W.E.L. , JunLin Liu, De-Hui Shiue, "Object Segmentation of Database Images by Dual Multiscale Morphological Reconstructions and Retrieval Applications", IEEE Transactions on Image Processing, Vol. 21, No. 2, Feb. 2012.

[20] Liu XS1, Shane E, McMahon DJ, Guo XE., "Individual trabecula segmentation (ITS)-based morphological analysis of microscale images of human tibial trabecular bone at limited spatial resolution", J Bone Miner Res., vol. 26 no. 9, pp. 2184-2193, Sep 2011. doi: 10.1002/jbmr.420

[21] Gillibert L1, Peyrega C, Jeulin D, Guipont V, Jeandin M., "3D multiscale segmentation and morphological analysis of X-ray microtomography from cold-sprayed coatings", Journal of Microscopy, vol. 248, no. 2, pp. 187-199, Nov 2012.
[22] A. Korzynska, M. Iwanowski, "Multistage morphological segmentation of bright-field and fluorescent microscopy images", Opto-Electronics Review, Vol. 20, no. 2, pp. 174-186, June 2012.

[23] Md. Motiur Rahman, Abdul Aziz, Mithun Kumar PK, Mohammad Abu Naim Uddin Rajiv and Mohammad Shorif Uddin, "An Optimized Speckle Noise Reduction Filter For Ultrasound Images using Anisotropic Diffusion Technique", International journal of Imaging, vol. 8, no. 2 2012.

[24] Yi Wang, Ruiqing Niu, Liangpei Zhang, Huanfeng Shen, "Region-based adaptive anisotropic diffusion for image enhancement and denoising", Optical Engineering, vol. 49, no. 11, November 2010.

[25] Xiaoming Liu, Jun Liu, Xin Xu, Lei Chun, Jinshan Tang, and Youping Deng, "A robust detail preserving anisotropic diffusion for speckle reduction in ultrasound images", BMC Genomics, 12(Suppl 5): S14, 2011.

[26] Mohammad Motiur Rahman, Mithun Kumar PK, Abdul Aziz, Md. Gauhar Arefin and Mohammad Shorif Uddin, "Adaptive anisotropic diffusion filter for speckle noise reduction for ultrasound images", International Journal of Convergence Computing, Vol. 1, No. 1, 2013.

[27] Shin-Min Chao, Du-Ming Tsai, Wei-Yao Chiu, Wei-Chen $\mathrm{Li}$, "Anisotropic diffusion-based detail-preserving smoothing for image restoration" 17th IEEE International Conference on Image Processing (ICIP), Hong Kong, 2010.

[28] Hum Yan Chai, Lai Khin Wee, Eko Supriyanto, "Edge detection in ultrasound images using speckle reducing anisotropic diffusion in canny edge detector framework", Proceedings of the 15th WSEAS international conference on Systems, pp. 226-231, Stevens Point, Wisconsin, USA, 2011.

[29] Boucher M., Evans A., Siddiqi K., "Anisotropic diffusion of tensor fields for fold shape analysis on surfaces", Springer-Proceedings of the 22nd International Conference on Information Processing in Medical Imaging, Canada, pp. 271-282, 2011.

[30] Qian Wu, Yu Chen, Gang Teng, "Lattice Boltzmann Anisotropic Diffusion Model Based Image Segmentation", Proceeding of the Computer, Informatics, Cybernetics and Applications (CICA)-Lecture Notes in Electrical Engineering Volume 107, pp 577-585, 2012.

[31] Jae Sung Lim, Sung In Cho, and Young Hwan Kim, "Accuracy enhancement of image segmentation using adaptive anisotropic diffusion", IEEE 2nd Global Conference on Consumer Electronics (GCCE), Tokyo, pp 451 - 452, 1-4 Oct. 2013. DOI: 10.1109/GCCE.2013.6664887

[32] Bi L, Kim J, Wen L, Kumar A, Fulham M, and Feng DD, "Cellular automata and anisotropic diffusion filter based interactive tumor segmentation for positron emission tomography", Conf. Proc. IEEE Eng. Med. Biol. Soc., pp. 5453-5456, 2013. doi: 10.1109/EMBC.2013.6610783.

[33] Bhagwati Charan Patel and G. R. Sinha, "Energy and Region based Detection and Segmentation of Breast Cancer Mammographic Images", IJIGSP, vol. 4, no. 6, pp.44-51, 2012. DOI: 10.5815/ijigsp.2012.06.07.

[34] Abo-Eleneen Z. A, Gamil Abdel-Azim, "A Novel Approach for MRI Brain Images Segmentation", IJIGSP, vol. 5, no. 3, pp. 10-18, 2013. DOI: 10.5815/ijigsp.2013.03.02.

[35] Atiyeh Hashemi, Abdol Hamid Pilevar, Reza Rafeh, "Mass Detection in Lung CT Images Using Region Growing Segmentation and Decision Making Based on 
Fuzzy Inference System and Artificial Neural Network", IJIGSP, vol. 5, no. 6, pp. 16-24, 2013.DOI: 10.5815/ijigsp.2013.06.03.

[36] Bergeest JP, and Rohr K., "Efficient globally optimal segmentation of cells in fluorescence microscopy images using level sets and convex energy functionals", Med Image Anal., vol. 16, no. 7, pp. 1436-44, Oct 2012. doi: 10.1016/j.media.2012.05.012.

[37] Rafika Harrabi,Ezzedine Ben Braiek, "Color image segmentation using multi-level thresholding approach and data fusion techniques: application in the breast cancer cells images", EURASIP Journal on Image and Video Processing, vol. 2012, no. 11, May 2012. DOI: 10.1186/1687-5281-2012-11.

[38] Sonu Kumar Jha, Purnendu Bannerjeeb, Subhadeep Banika, "Random Walks based Image Segmentation Using Color Space Graphs", Procedia Technology,Vol. 10, pp. 271-278, 2013.

[39] Miranda, P.A.V., Falcao, A.X., and Spina, T.V., "Riverbed: A Novel User-Steered Image Segmentation Method Based on Optimum Boundary Tracking", IEEE Transactions on Image Processing, vol. 21, no. 6, pp. 3042 - 3052, June 2012. DOI: 10.1109/TIP.2012.2188034.

[40] Merciol, F., and Lefevre, S., "Fast Image and Video Segmentation Based on Alpha-tree Multiscale Representation", IEEE Eighth International Conference on Signal Image Technology and Internet Based Systems (SITIS), pp. 336 - 342, Naples, 25-29 Nov. 2012. DOI: 10.1109/SITIS.2012.56.

[41] W. J. Niessen, K. L. Vincken, J. A. Weickert, and M. A. Viergever, "Nonlinear multiscale representations for image segmentation," Computer Vision and Image Understanding, vol. 66, pp. 233-245, 1997.

[42] D. Ghoshal and P. P. Acharjya, “ Effect of Various Spatial Sharpening Filters on the Performance of the Segmented Images using Watershed Approach based on Image Gradient Magnitude and Direction", International Journal of Computer Applications (0975 - 8887), Vol. 82, No. 6, November 2013.

[43] Angulo, J., Velasco-Forero, S., "Semi-supervised hyperspectral image segmentation using regionalized stochastic watershed", in: Proceedings of SPIE symposium on Defense, Security, and Sensing: Algorithms and Technologies for Multispectral, Hyperspectral, and Ultraspectral Imagery XVI, SPIE, Bellingham. p. 76951F, 2010.

[44] K. B. Bernander1, K. Gustavsson1, B. Selig, I. Sintorn, C. L. L. Hendriks, "Improving the Stochastic Watershed", Pattern Recognition Letters, Vol. 34, No. 9, pp. 993-1000, July 2013.

\section{Authors' Profiles}

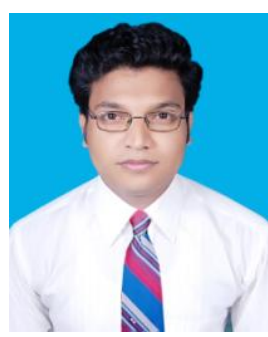

Mithun Kumar PK was born in Rajshahi, Bangladesh in 1989. He received the B.Sc Engineering degree in Computer Science \& Engineering from Mawlana Bhashani Science and Technology University, Santosh, Tangail, Dhaka, Bangladesh, in 2012.

$\mathrm{He}$ is currently working as a RESEARCHER with international research teams. He has approximately five years experience in digital image processing and medical image processing. His research interests include image analysis, image processing \& medical image processing, pattern recognition, 3D visualization, Segmentation, Filter Optimization etc.

Mr. PK's has many international journal and conference publications all over the world. Now, he is a regular reviewer at IET Image Processing journal, Journal of Media and Communication studies, and International Arab Journal of Information Technology.

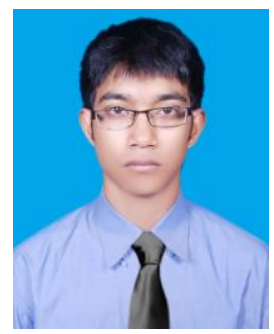

Md. Gauhar Arefin was born in Nilphamari, Bangladesh in 1990. He received the B.Sc Engineering degree in Computer Science \& Engineering from Mawlana Bhashani Science and Technology University, Santosh, Tangail, Dhaka, Bangladesh, in 2012.

Now, he is working as a SOFTWARE ENGINEER at a software company in Dhaka, Bangladesh. He has about one and half years experience in Software Company and five years experience in image processing. His research interests include image analysis, image processing \& medical image processing, 3D visualization.

Mr. Arefin's has many international journal and conference publications all over the world.

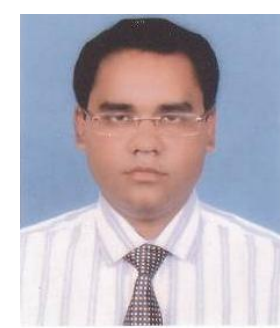

Mohammad Motiur Rahman was born in Gafargaon, Dhaka, Bangladesh, in $1^{\text {st }}$ September, 1973 and received the B.Sc Engg. \& M.S degree in Computer Science \& Engineering from Jahangirnagar University, Dhaka, Bangladesh, in 1995 \& 2001, where he is currently pursuing the Ph.D. degree.

$\mathrm{He}$ is currently working as an ASSOCIATE PROFESSOR at Computer Science \& Engineering department in Mawlana Bhashani Science and Technology University, Santosh, Tangail, Dhaka, Bangladesh. He joined in this department as a lecturer in 2003 and after three years, he worked in this same department as an assistant professor from 2006 to 2013. He has about ten years experience in both teaching and research. His research interests include digital image processing, medical image processing, computer vision, data mining \& digital electronics.

Prof. Rahman's has many international journal and conference publications all over the world.

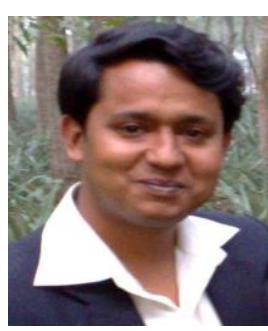

A. S. M. Delowar Hossain was born in Manikganj, Dhaka, Bangladesh and received the B.Sc Engg. \& M.S degree in Computer Science \& Engineering from Islamic University of Technology (IUT), Gazipur, Dhaka, Bangladesh.

$\mathrm{He}$ is currently working as an ASSISTANT PROFESSOR at Computer Science \& Engineering department in Mawlana Bhashani Science and Technology University, Santosh, Tangail, Dhaka, Bangladesh. He joined in this department as a lecturer and after three years, he is working in this same department as an assistant professor. He has about seven years experience in both teaching and research. His research interests include data mining, cloud computing, embedded operating system, digital image processing and computer vision.

Prof. Hossain's has many international journal and conference publications all over the world. 